\title{
A 3-D Finite Element Approach for Predicting the Transmission Loss in Mufflers and Silencers with no Mean Flow
}

\author{
Omid Z. Mehdizadeh ${ }^{1}$, Marius Paraschivoiu ${ }^{2 *}$ \\ ${ }^{1}$ Department of Mechanical and Industrial Engineering, University of Toronto, 5 King 's College Circle, \\ Toronto, Ontario,omidzm@mie.utoronto.ca \\ ${ }^{2}$ Department of Mechanical and Industrial Engineering, Concordia University, 1455 de Maisonneuve Blvd. \\ West, Montreal, Quebec, paraschi@me.concordia.ca
}

*Corresponding author

A three-dimensional finite element method has been implemented to predict the transmission loss of a packed muffler and a parallel baffle silencer for a given frequency range. Iso-parametric quadratic tetrahedral elements have been chosen due to their flexibility and accuracy in modeling geometries with curved surfaces. For accurate physical representation, perforated plates are modeled with complex acoustic impedance while absorption linings are modeled as a bulk media with a complex speed of sound and mean density. Domain decomposition and parallel processing techniques are applied to address the high computational and memory requirements. The comparison of the computationally predicted and the experimentally measured transmission loss shows a good agreement.

Keywords: finite element method, Helmoltz equation, Tetrahedron elements

\section{INTRODUCTION}

Since numerous physical phenomena include a form of wave propagation, there has always been an interest in understanding and modeling wave propagation and its interactions with other physical phenomena.

In general, there are three concerns in developing an appropriate model for a physical phenomenon: the complexity of the actual physical phenomenon, the minimum required accuracy and lastly, the available analytical, experimental and computational resources. Due to the fact that phenomena involving wave propagation can potentially be complex and require high accuracy, this constraint in computational resources limits the models to address rather simple problems only. However, in recent years, swift advances in the computational capacity of microprocessors and lower prices for memory have created a new perspective for developing other powerful models, such as finite element methods, to address complex wave propagation problems.

This work focuses primarily on noise control technology. A three-dimensional finite element method is implemented and applied to solve several noise control problems. 
More specifically, three-dimensional time-harmonic wave propagation in air and porous media is modeled whereby porous materials may be used as acoustic absorbers or as filters in mufflers and silencers. Therefore, finite element modeling can be used for predicting the transmission loss $(T L)$ in the problem of interest at a given frequency range.

In the last decade, finite element methods have been widely used to solve Helmholtz's equation, a governing equation for time-harmonic wave propagation, mainly in twodimensional domains. A major challenge for using finite element methods for Helmholtz's equation is that a specific resolution requirement for minimal wavelength must be respected for control of the approximation error. Dispersion analysis demonstrates that because of a pollution effect associated with a phase error, it is generally more difficult to meet the resolution requirements for higher frequencies [1]. In one of the most recent studies, general resolution rules that account for the pollution effect are derived by F. Ihlenburg [2].

Several finite element methods have been developed to ease resolution requirements which are seemingly an open problem. C. Farhat et al. [3] briefly reviewed many suggested methods in the literature and, consequently, offered a discontinuous Galerkin method with plane wave basis functions as the most effective approach for solving short wave Helmholtz's problems. A simpler modification of the standard Galerkin finite element method based on least-squares stabilization is provided by I. Harari et al. [4] to effectively relax the resolution requirements. Since the focus of this work is on the application of the finite element method, the use of quadratic elements are deemed sufficiently capable of addressing this issue.

As mentioned before, due to restrictions in computational resources, most of the early published applications of finite element methods for time-harmonic wave propagation had been limited to two-dimensional domains. This has been changed in recent years, and more examples of three-dimensional applications can be found in the literature. For instance, a three-dimensional finite element model was developed by T. Koike et al. [5] to clarify the acoustic mechanisms of the human middle ear without direct measurements since these are difficult to conduct. R. Tezaur et al. [6] generated a three-dimensional finite element method with quadratic tetrahedral elements for acoustic scattering problems in exterior domains. The acoustic absorption of multi-layer absorbers was studied by F. -C. Lee et al. [7] using a Galerkin finite element method with eight-node brick elements.

While recent versions of most commercially available finite element software packages, such as FEMLAB, ABAQUS, MSC.Nastran and ANSYS, have included threedimensional acoustic simulations, it is still advantageous to develop an open-source finite element code because many improvements to the numerical methods and the physical modeling remain. In this work, a particular parallel processing concept has been implemented to address larger problems with the available resources. Furthermore specific components of mufflers and silencers, such as perforated plates and absorbing materials have been modeled. 
Regarding applications for mufflers and silencers, M. L. Munjal [8], in an overview of the last decade of research in the Indian Institute of Science, concluded that more research is required in "FEM analysis of complex geometries like perforated element mufflers in order to incorporate 3-D effects". In a recent study, S. Bilawchuk et al. [9] compared various numerical methods for calculating the transmission loss in silencers. It was concluded that the combination of the finite element method and the three-point method is advantageous over other considered methods. A three-dimensional direct mixed-body boundary element method for packed silencers was created by $\mathrm{T}$. W. Wu et al. [10], including modeling of perforated plates and two different acoustic media; i.e. air and absorbing material. This method is based on the multi-domain boundary element method which necessitates homogeneous and isotropic subdomains. To verify the formulation, several test cases were examined and the results were compared with experimental data. In this work, we examine several of those test cases using our threedimensional finite element method.

In practice, finite element simulation requires high computational and memory resources, particularly at higher frequencies. Using domain decomposition and parallel processing techniques, one can take advantage of the simultaneous computational and memory capacity of several computers for solving one problem in parallel. Our methodology applies a domain decomposition method for division of the original system into several subproblems.

Following this introduction, diverse general noise control problems, including the problems at hand, will be described in Section 2. Section 3 briefly presents mathematical models of wave propagation in an ideal gas and porous media, as well as through a perforated plate. In Section 4, several common approaches to investigate noise control problems will briefly be reviewed and a finite element method is presented in more detail. Section 5 reports the finite element results and provides a comparison with experimental data. Finally, Section 6 will conclude this work and suggest future research directions.

\section{Noise Control Problems}

There are a variety of noise sources and noise propagation problems surrounding us. However, almost all noise propagation problems belong to one of the two main categories. The first category involves noise propagation problems whereby the frequency and the amplitude of the source are known; for example, a vibrating structure or fluid on the boundary of the domain. The second type entails noise propagation problems such that the source of the noise is coupled with the noise propagation problem. An example of this is the turbulence of fluid flow within the domain. These two main categories of noise control problems are known as, respectively, vibroacoustic and aeroacoustic problems. For the problems we examine, the major source of noise is known at the inlet pipe; hence, these problems were considered as vibroacoustic problems. 
Generally speaking, the goal is to reduce noise since excessive noise in our environment is shown to have damaging effects on human beings and other living creatures. Furthermore, high amplitude noise may produce vibrations and this can consequently lead to fatigue and the eventual break down of machine parts.

Passive noise control techniques, such as absorption panels, acoustic enclosures, double glaze windows, mufflers, silencers and earplugs, are remedies implemented for controlling the impact of noise. A more recent approach in noise control technology is known as active noise control. It is based on measuring noise or vibration and then producing a wave with almost the same amplitude but in opposite phase in order to cancel out original noise or vibration. This is a relatively expensive process since it requires the use of microphones or accelerometers, a real-time control system and speakers or actuators.

Accurate modeling of an acoustic field is an essential part of the design procedure in noise control systems. In this study, a packed muffler and a parallel baffle silencer are chosen as examples of noise control devices. Mufflers and silencers are extensively used in inlets and outlets of internal combustion engines, air compressors and fans for reducing the propagation of the noise generated in those machines.

The most important acoustic property of a muffler and a silencer is its transmission loss which is defined as the difference between the output and input noise amplitude for a given frequency. The traditional method for evaluating the $T L$ requires the solution of the acoustic field for two different boundary conditions and is called the four-pole method [11]. However, described in this research work is a faster method, the three-point method [12], used for the evaluation of the $T L$.

\section{Three-Point Method}

In the three-point method, the $T L$ can be calculated with a single solution for each frequency. This setting is presented in figure 1 . At the input, there is a uniform velocity (or pressure) and the output is assumed anechoic. Letting $x_{1}$ and $x_{2}$ represent the coordinates of two points along the muffler axis while $p$, generally a complex value, represents the amplitude of the sound pressure at each point, the $T L$ can therefore be evaluated by means of the following equation:

$T L=20 \log _{10} \frac{\left|p_{i}\right|}{\left|p_{3}\right|}+10 \log _{10} \frac{S_{i}}{S_{o}}$,

where $S_{i}$ and $S_{o}$ are the cross-sectional areas of the inlet and outlet tubes respectively. Furthermore:

$$
p_{i}=\boldsymbol{\zeta}_{1} e^{i k x_{2}}-p_{2} e^{i k x_{1}} \geqslant\left(i \sin \mid \boldsymbol{C}_{2}-x_{1}\right) \text {, }
$$

and

$$
\begin{aligned}
& \left|p_{i}\right|=\left\{p_{\mathrm{Re} 1}^{2}+p_{\mathrm{Im} 1}^{2}+p_{\mathrm{Re} 2}^{2}+p_{\mathrm{Im} 2}^{2}-2\left(p_{\mathrm{Re} 1} p_{\mathrm{Re} 2}+p_{\mathrm{Im} 1} p_{\mathrm{Im} 2}\right) \cos \backslash \mathbb{4}_{2}-x_{1}-\right. \\
& \left.-2\left(p_{\mathrm{Re} 1} p_{\mathrm{Im} 2}-p_{\mathrm{Im} 1} p_{\mathrm{Re} 2}\right) \sin \left|\boldsymbol{4}_{2}-x_{1} \bar{\jmath} /\right| 2 \sin \mid \boldsymbol{4}_{2}-x_{1}\right]
\end{aligned}
$$


If $c$ is the speed of sound propagation in the fluid and $\omega$ is the angular frequency, then $k$ is the wave number defined as $k=\omega / c$. Subscripts $\mathrm{Re}$ and $\mathrm{Im}$ denote the real part and the imaginary part of a complex variable.

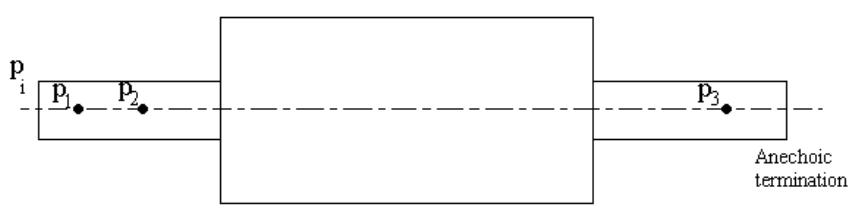

Figure 1: Location of the Points for the Three-Point Method in a Muffler

\section{Mathematical Modeling}

In aeroacoustic studies, the acoustic field is coupled with the flow field. For compressible flow, the main governing equations are a set of non-linear partial differential equations: the Navier-Stokes equations. In vibroacoustic studies, the acoustic field may be coupled with vibrations of the solid structure. Therefore, the governing equation is the wave equation in the elastic medium, which is linear. However, in most cases, the acoustic pressure is not strong enough to have a substantial impact on the solid structure so the two fields are not coupled. It is often the case that, in practice, the wave equation in an elastic solid is solved for the structure. Then, the solution enables one to determine the boundary conditions for the wave equation in the fluid.

The wave equation for a perfect gas with no damping can be derived from three basic fluid dynamic equations [11]. The linear wave equation is presented in equation (10).

$$
\frac{\partial^{2} P}{\partial t^{2}}=c^{2} \nabla^{2} P
$$

where $P$ is the sound pressure and $c$ is the speed of sound such that $c^{2}=\left.\frac{\partial P}{\partial \rho}\right|_{\text {isentropic }}$.

Solving equation (10) with the corresponding boundary and initial conditions in the time domain gives $P$ as a function of time and space.

By assuming a time-harmonic solution for the sound pressure, $P=p e^{i \omega t}$, the linear wave equation reduces to Helmholtz's equation given as equation (11):

$\nabla^{2} p=-k^{2} p$.

It should be noted that $p$ is in the frequency domain and therefore has a complex value.

For mufflers and silencers, the transient state is not important so the acoustic field can be solved by applying Helmholtz's equation at every frequency.

\section{Perforated Plate}


The basic assumptions for modeling a perforated plate in an acoustic field are continuous normal velocity and discontinuous pressure through the plate. Several empirical relations exist for relating the normal velocity, $u_{n}$, and the pressure jump , $\Delta p$, through a perforated plate for various conditions. The simple relation proposed by Sullivan and Crocker [13] is considered sufficiently accurate for usage in this report. The dimensionless transfer impedance $Z_{t}$ of a perforated plate can be approximated as follows:

$$
Z_{t}=\frac{1}{\rho_{0} c \sigma} \text { e. } 4+i 0.02 f^{-}
$$

where $\sigma$ is the ratio of the open area to the total area of the plate. The pressure jump can be calculated as designated here below:

$$
\Delta p=\rho_{0} c Z_{t} u_{n} \text {. }
$$

Also, the normal particle velocity, $u_{n}$, and the normal sound pressure gradient are related as demonstrated in equation (14):

$$
\frac{\partial p}{\partial n}=-i \rho_{0} \omega u_{n} \text {. }
$$

\section{Porous Media}

Sound absorbing materials are exploited to absorb acoustic energy which eventually converts itself into thermal energy due to viscous and thermal effects. Sound absorbing materials are generally highly porous materials, so the acoustic wavelength is generally much greater than the dimensions of the pores. This is the key point in modeling porous media as a bulk. Based on the relations developed by Delany and Bazley [14,15], a porous material can be replaced by an equivalent fluid that is characterized by a complex speed of sound, $c_{B}$, and a complex mean density, $\rho_{B}$. Both $c_{B}$ and $\rho_{B}$ are related to the propagation constant, $\Gamma_{B}$, and the characteristic impedance, $Z_{B}$, by these equations:

$c_{B}=\frac{i \omega}{\Gamma_{B}}$,

$\rho_{B}=\frac{Z_{B}}{c_{B}}$.

$\Gamma_{B}$ and $Z_{B}$ can be measured directly by the two-cavity method [16] or they can be calculated by empirical power law approximations [17], provided that the flow resistivity, $R$, is known.

\section{APPROACHES FOR MUfFler ANALysiS}

One of the first approaches utilized for studying mufflers referred to one-dimensional analysis in the frequency domain based on electro-acoustic analogy [18]. This approach has since then been elaborated on and combined with empirical approximations to deal with the effects of perforated elements, mean flow and mean temperature gradient [19, 20, 21, 22]. Another one-dimensional approach employed is based on one-dimensional analysis in the time domain. In the latter approach, the equations are solved numerically in the time-domain to evaluate all acoustic waves simultaneously. One-dimensional analyses, based on plane-wave analysis, can predict muffler performance accurately as long as three-dimensional effects are ignorable. Hence, these analyses tend to be less 
accurate when dealing with higher frequencies and complicated geometries. This can quite possibly be the reason why, in the past, the final design for mufflers was achieved mostly by trial and error. Moreover, designers were also limited to a certain number of basic elements, such as perforated tubes and expansion chambers, which were available and examined throughout numerous years.

In the last decade, however, three-dimensional numerical analyses of mufflers, such as boundary element and finite element, have proved to be powerful tools for more accurate performance prediction and muffler designs. Three-dimensional numerical analyses require high computational and memory resources. Boundary element methods, BEMs, have the advantage of performing computations only on the boundaries of the domain and consequently, these methods have been applied to three-dimensional analysis more often than other available methods. However, finite element methods, FEMs, perform computations on the entire domain and are therefore more powerful. More specifically, FEMs are able to address problems in non-homogeneous domains. So, they have the potential to address acoustic problems in the presence of absorption materials, mean flow and mean temperature gradient. Moreover, it is shown that FEMs compare favorably to BEMs with regards to computational costs [23].

\section{The Finite Element Method}

In this paper, a three-dimensional finite element method is implemented to assess the $T L$ in a packed muffler and a parallel baffle silencer as illustrated in figure 2 and figure 3 , respectively. In the parallel baffles, the absorption material is separated from air by keeping perforated plates on the sides and plane plates at the two ends. These examples are chosen to show the flexibility and accuracy of the method in addressing problems that arise when implementing typical components of a muffler or silencer. They are also selected due to the fact that measurement data is available [10]. It should be noted, however, that the application of the finite element method is not limited to these cases only.

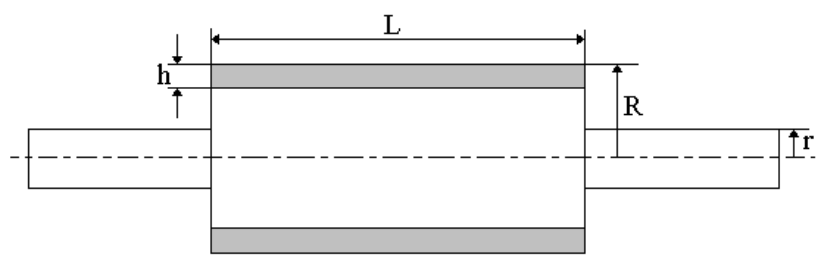

Figure 2: A Packed Muffler with Polyester Lining 


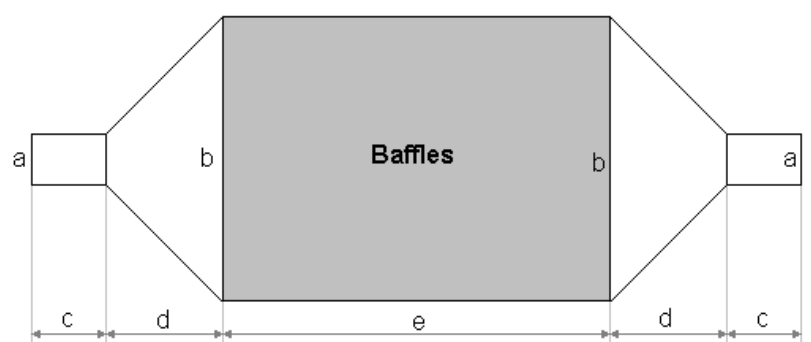

(a)

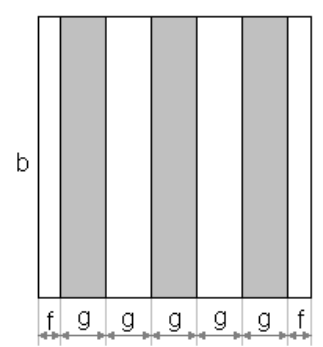

(b)

Figure 3: A Parallel Baffle Silencer; (a) Exterior Geometry, (b) Cross Section of the Parallel Baffles

The governing equations can be written as shown below:

$$
\begin{aligned}
& \nabla^{2} p_{a}+k_{a}^{2} p_{a}=0 \text { in } \Omega_{a}, \\
& \nabla^{2} p_{B}+k_{B}^{2} p_{B}=0 \text { in } \Omega_{B},
\end{aligned}
$$

where $\Omega_{a}$ and $\Omega_{B}$ are the domains of air and bulk porous material, respectively. The boundary conditions are consequently:

$\frac{\partial p}{\partial n}=0$ on rigid walls,

$p=1$ at the inlet,

$\frac{\partial p}{\partial n}=i k p \quad$ at the outlet.

One-dimensional plane-wave propagation is assumed at the inlet and the outlet tubes. Hence, the anechoic boundary condition at the outlet has a rather simple form of a Robin boundary condition, identified in equation (20). At the interface between air and porous material, the normal velocity is continuous and the normal pressure gradient is proportional to the density ratio, thus represented as equation (21):

$$
u_{a} \cdot n_{a}=-u_{B} \cdot n_{B}, \quad \frac{\partial p_{B}}{\partial n}=-\frac{\rho_{B}}{\rho_{a}} \frac{\partial p_{a}}{\partial n} .
$$

On the perforated plate, equation (13) relates the two sides of the plate. Note that equation (12) is valid only for a perforated plate surrounded by air, so we must assume a narrow air gap between the absorption material and the perforated plate.

At this point, one must introduce the following continuous space of complex functions: 


$$
Z=\not{h}=v_{\mathrm{Re}}+i v_{\mathrm{Im}}: v_{\mathrm{Re}} \in H^{1}(\Omega), v_{\mathrm{Im}} \in H^{1}(\Omega),
$$

where $H^{1}$ is the Hilbert space. The variational formulation of the problem is to find $p \in Z$ such that:

$$
\begin{aligned}
& \int_{\Omega_{a}} \nabla v \cdot \nabla p_{a}-k_{a}^{2} v p_{a} \bar{d} \Omega+\int_{\Omega_{B}} \frac{\rho_{a}}{\rho_{B}} \nabla v \cdot \nabla p_{B}-k_{B}^{2} v p_{B} \frac{\partial}{d} \Omega+\int_{S_{p 1}} i \frac{k_{a}}{Z_{t}} v \boldsymbol{\zeta}_{p 1}-p_{p 2} \bar{d} S \\
& +\int_{S_{p 2}} i \frac{k_{a}}{Z_{t}} v \boldsymbol{\zeta}_{p 2}-p_{p 1} \underset{d}{d} S-\int_{S_{o}} i k_{a} v p_{o} d S=0 \quad \forall v \in Z
\end{aligned}
$$

where $S_{o}$ is the cross-sectional area of the output pipe and $S_{p 1}$ and $S_{p 2}$ are surfaces on both sides of a perforated plate. In equation (23), the first two terms (volume integrals) are associated with the governing equations for air and the porous material, respectively. The next two terms (surface integrals) are associated with the pressure jump through the perforated plates and they link the pressure of the two sides of the perforated plates. The last term (a surface integral) is associated with the Robin boundary condition which can model the anechoic termination of the outlet tube. The computational model utilized is the standard Galerkin finite element method. The entire three-dimensional domain, $\Omega$, is divided into $K$ conforming non-overlapping tetrahedral elements, $\hat{\Omega}_{j}$, such that:

$$
\bar{\Omega}=\bigcup_{j=1}^{K} \bar{\Omega}_{j} \text {. }
$$

Iso-parametric quadratic elements are applied to model curved surfaces more accurately. More importantly, higher order elements are generally more accurate for wave problems. This has been shown both by dispersion analysis $[1,31]$ and by by numerical experiments [32].

The discrete approximation space, $H_{h}^{1} \subset H^{1}$, is defined as:

$$
H_{h}^{1}=\hat{h}=v_{\mathrm{Re}}+i v_{\mathrm{Im}}: v_{\mathrm{Re}} \in \mathrm{P}_{2}\left(\hat{\Omega}_{j}\right), v_{\mathrm{Im}} \in \mathrm{P}_{2}\left(\hat{\Omega}_{j}\right) \text {, }
$$

where $\mathrm{P}_{2}$ are polynomials of degree two defined on each tetrahedral element, $j$, with four nodes on the corners and six nodes at the midpoint of each edge.

The global basis functions, $\phi_{n} \in H_{h}^{1}$, are defined as:

$$
\phi_{n}\left(X_{m}\right)=\delta_{n m}, \quad 1 \leq n, m \leq N \text {, }
$$

where $N$ is the total number of global nodes and $X$ is the coordinates of a global node.

Using global basis functions, any function, $v$, can be approximated as:

$$
v(X) \approx v_{h}(X)=\sum_{m=1}^{N} v_{m} \phi_{m}(X),
$$

where $v_{m}=v\left(X_{m}\right)$.

Using equation (27) to expand all the functions appearing in the variational formulation, (equation (23)) in the discrete approximation space (equation (25)), the discretized equation leads to a linear system of algebraic equations obtained here below:

$\mathbf{A p}=\mathbf{f}$, 
where the coefficient matrix $\mathbf{A}$ is a sparse symmetric matrix, $\mathbf{p}$ is the vector of nodal values of the sound pressure amplitude and $\mathbf{f}$ includes the vector of nodal values of the forcing function. Here, $\mathbf{f}$ is non-zero only due to non-homogeneous Dirichlet boundary conditions at the inlet pipe.

Since $\mathbf{A}, \mathbf{p}$ and $\mathbf{f}$ have complex values the system can be written as:

$$
\left(\mathbf{A}_{\mathrm{Re}}+i \mathbf{A}_{\mathrm{Im}}\right)\left(\mathbf{p}_{\mathrm{Re}}+i \mathbf{p}_{\mathrm{Im}}\right)=\mathbf{f}_{\mathrm{Re}}+i \mathbf{f}_{\mathrm{Im}} \text {. }
$$

As a block system of equations, equation (29) corresponds to:

$$
\left\{\begin{array}{l}
\mathbf{A}_{\mathrm{Re}} \mathbf{p}_{\mathrm{Re}}-\mathbf{A}_{\mathrm{Im}} \mathbf{p}_{\mathrm{Im}}=\mathbf{f}_{\mathrm{Re}} \\
\mathbf{A}_{\mathrm{Im}} \mathbf{p}_{\mathrm{Re}}+\mathbf{A}_{\mathrm{Re}} \mathbf{p}_{\mathrm{Im}}=\mathbf{f}_{\mathrm{Im}}
\end{array} .\right.
$$

The block system given as equation (29) can be rewritten as a symmetric system:

$$
\left[\begin{array}{cc}
\mathbf{A}_{\mathrm{Re}} & \mathbf{A}_{\mathrm{Im}} \\
\mathbf{A}_{\mathrm{Im}} & -\mathbf{A}_{\mathrm{Re}}
\end{array}\right]\left[\begin{array}{c}
\mathbf{p}_{\mathrm{Re}} \\
-\mathbf{p}_{\mathrm{Im}}
\end{array}\right]=\left[\begin{array}{l}
\mathbf{f}_{\mathrm{Re}} \\
\mathbf{f}_{\mathrm{Im}}
\end{array}\right] \text {. }
$$

The block system derived above is solved using a parallel conjugate gradient iterative solver with a localized ILU(0) preconditioner that has no global dependency. An additive Schwarz domain decomposition method (ASM) for overlapped regions is applied to stabilize the localized ILU(0) preconditioning. Details of the domain decomposition and preconditioning technique can be found in the work of K. Nakajima and H. Okuda [24]. The standard Message Passing Interface (MPI) [25] subroutines are called in the code for all the message passing operations between the processors.

While solving the above real system by a preconditioned conjugate gradient solver shows acceptable convergence for this particular problem, it should be noted this does not necessarily hold for general complex systems arising from finite element discretizations of Helmholtz's equation because the resulting system is not always positive definite. Several iterative solvers for such complex systems have been developed and examined for different problems [28, 29, 30].

The components of the coefficient matrix $\mathbf{A}, A_{m n}$, are:

$$
\begin{aligned}
& A_{m n}=\int_{\Omega_{a}} \nabla \phi_{m} \cdot \nabla \phi_{n}-k_{a}^{2} \phi_{m} \phi_{n} \bar{d} \Omega+\int_{\Omega_{B}} \frac{\rho_{a}}{\rho_{B}} \boldsymbol{\nabla} \phi_{m} \cdot \nabla \phi_{n}-k_{B}^{2} \phi_{m} \phi_{n} \bar{d} \Omega+ \\
& \int_{S_{p 1}} i \frac{k_{a}}{Z_{t}} \phi_{m} \boldsymbol{\phi}_{n}-\phi_{n} \underline{d} S+\int_{S_{p 2}} i \frac{k_{a}}{Z_{t}} \phi_{m} \boldsymbol{\phi}_{n}-\phi_{n} \underline{d} S-\int_{S_{o}} i k_{a} \phi_{m} \phi_{n} d S, \quad 1 \leq m, n \leq N
\end{aligned}
$$

When applying equation (33), each component is first evaluated on each element, and then elemental matrices are assembled to form the global matrix $\mathbf{A}$. In order to perform operations such as integration and differentiation required for calculating the elemental matrices, each element is transformed from the global coordinate system $(x, y, z)$ to the reference elemental coordinate system $\left(\xi_{1}, \xi_{2}, \xi_{3}\right)$.

For quadratic iso-parametric tetrahedral elements, the coordinates of the reference element are functions of the elemental basis functions, $h_{i}\left(\xi_{1}\right)$. The definition of the basis 
functions as well as the details of the quadratic iso-parametric elements can be found in most standard finite element text books [33, 34, 35].

The components of the elemental matrix $\hat{\mathbf{A}}, \hat{A}_{i j}$, for an interior element, $\hat{\Omega}$, can be presented as:

$$
\begin{aligned}
\hat{A}_{i j}= & \int_{\hat{\Omega}} \frac{1}{|J|}\left[\left(\sum_{l=1}^{3} J \frac{\partial h_{i}}{\partial \xi_{l}} \frac{\partial \xi_{l}}{\partial x}\right)\left(\sum_{l=1}^{3} J \frac{\partial h_{j}}{\partial \xi_{l}} \frac{\partial \xi_{l}}{\partial x}\right)+\left(\sum_{l=1}^{3} J \frac{\partial h_{i}}{\partial \xi_{l}} \frac{\partial \xi_{l}}{\partial y}\right)\left(\sum_{l=1}^{3} J \frac{\partial h_{j}}{\partial \xi_{l}} \frac{\partial \xi_{l}}{\partial y}\right),\right. \\
& \left.+\left(\sum_{l=1}^{3} J \frac{\partial h_{i}}{\partial \xi_{l}} \frac{\partial \xi_{l}}{\partial z}\right)\left(\sum_{l=1}^{3} J \frac{\partial h_{j}}{\partial \xi_{l}} \frac{\partial \xi_{l}}{\partial z}\right)\right] d \hat{\Omega}-\hat{k}^{2} \int|J| h_{i} h_{j} d \hat{\Omega}, \quad 1 \leq i, j \leq 10
\end{aligned}
$$

It should be highlighted that Jacobian, $J$, is not constant within an iso-parametric element.

Elemental integration is performed numerically using Gaussian quadrature defined on the reference element. Numerical integration leads to an exact result if the number of quadrature points is selected properly according to the order of the polynomial that is to be integrated. For instance, a fifth degree polynomial is integrated exactly with 15 integration points [26]. The surface integrations appearing in equation (33) are similarly performed on a two-dimensional triangular reference element.

\section{Results}

The packed muffler is a cylindrical expansion chamber with polyester lining, as shown in figure 2 . The radius of the inlet and outlet tubes is $r=2.54 \mathrm{~cm}(1 \mathrm{in}$.$) whereas the length$ and the radius of the chamber are $L=45.72 \mathrm{~cm}(18 \mathrm{in}$.) and $R=10.16 \mathrm{~cm}$ (4 in.), respectively. Also, the thickness of the polyester lining is $h=2.54 \mathrm{~cm}$ (1 in.). The parallel baffle silencer with three polyester baffles is shown in figure 3 . The dimensions shown in figure 3 are: $a=12.7 \mathrm{~cm}(5$ in. $), b=(24$ in. $), c=(6$ in. $), d=(12$ in. $), e=(36$ in. $), f=(2$ in. $)$ and $g=(4$ in. $)$. The polyester lining material has a flow resistivity equal to $16000 \mathrm{MKS}$ rayls/m.

The frequency range of interest is between 100 and $2800 \mathrm{~Hz}$. The element size for the finite element domain was chosen to provide a minimum resolution of 12 elements per wavelength to ensure that the resolution requirements were met and, consequently, that accuracy was maintained. Figures 4 and 5 show the discretized computational domain for the muffler and the silencer, respectively. In these figures, the curved surfaces appear as flat surfaces due to graphical limitations of the software. 


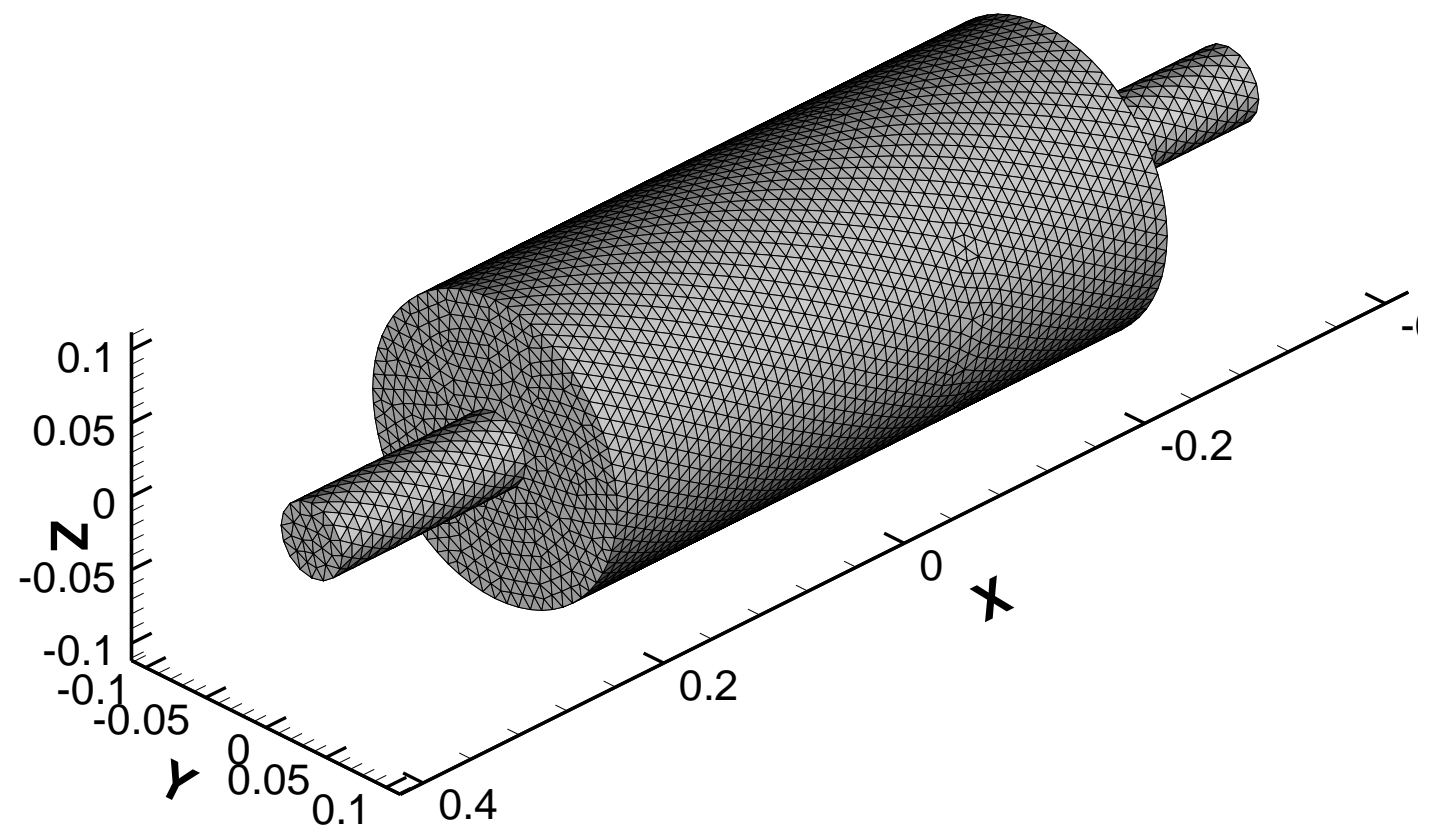

Figure 4: Finite Element Domain for the Packed Muffler: Surface Mesh

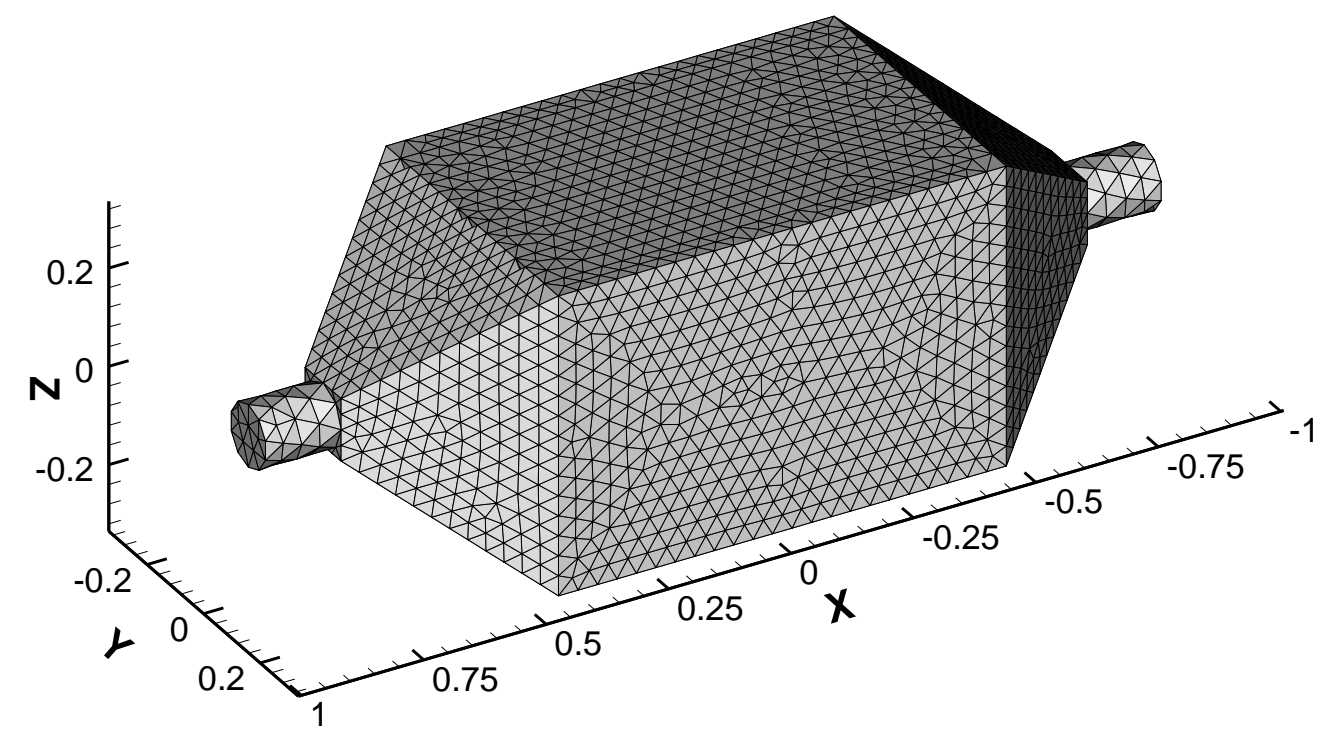

Figure 5: Finite Element Domain for the Parallel Baffle Silencer: Surface Mesh

In figure 6 and 7, the finite element results are reported and compared to the experimental and BEM results given by $\mathrm{T}$. W. Wu et al. [10]. Comparison of the results with the experimental data indicated that our finite element results are accurate for predicting the $T L$ in the frequency range of interest. The slight difference between the finite element results and the experimental data can be as a result of the mathematical modeling. As presented in section 3, the mathematical model assumes no absorption in the air and a perfect reflection on the walls; this is not accurate, especially at higher frequencies where an acoustic wave carries more energy. Moreover, the porous material and the perforated 
plates are approximated by complex acoustic impedance evaluated using rather simple empirical relations. This may explain the higher error in the results obtained for the silencer. As passive devices, acoustic damping in silencers is absorbed by material which ultimately plays a major role in the overall performance of the device. In mufflers, as reactive devices, impedance mismatch due to the sudden expansion is generally the key mechanism for suppressing noise.

In order to show the importance of modeling the lining porous material as an absorbing bulk rather than an absorbing surface, the results of the BEM with the local-reacting approach is also presented as figure 6. In the local reacting approach, the local impedance of the lining was measured and the chamber radius was set to $R^{*}=R-h$, where the local impedance is applied. As shown in figure 6, the boundary element results implementing the local-reacting approach are less accurate compared to the finite element results and boundary element results using bulk-reacting approach.

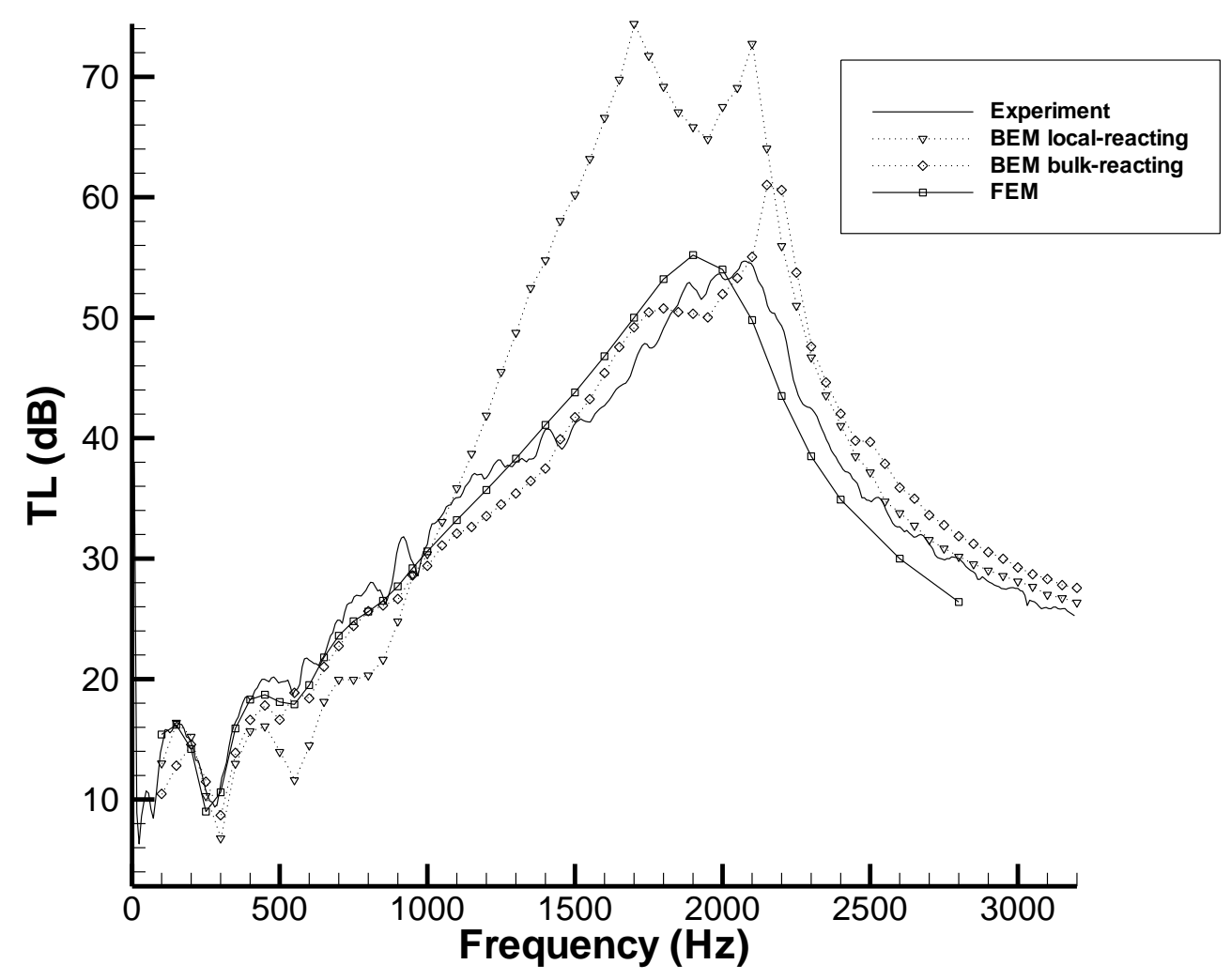

Figure 6: Comparison of TL for a Packed Muffler; Experiment and BEM (LocalReacting Approach) From T. W. Wu et al. [10]

In figure 7, the FEM results as well as the BEM results with the bulk-reacting approach are presented and they are compared to each other and with the experimental results. It is shown that the FEM results are similar to the BEM results and that both are in good agreement with the experimental results for most frequencies. The slight difference 
between the FEM and BEM results may be due to the difference in empirical relations applied for modeling the porous material.

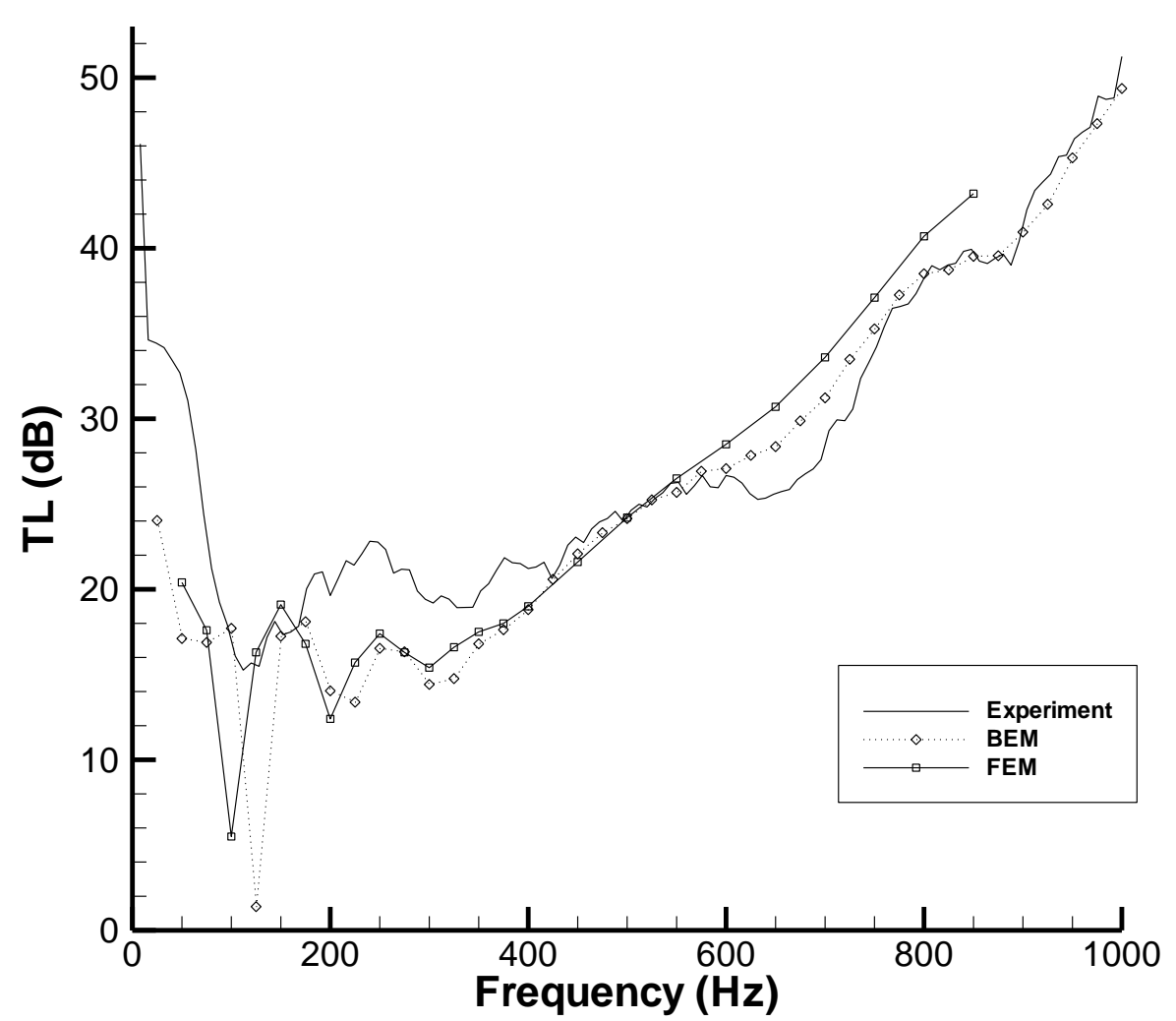

Figure 7: Comparison of TL for a Parallel Baffle Silencer; Experiment and BEM (BulkReacting Approach) From T. W. Wu et al. [10]

The frequency range for the silencer was limited to $800 \mathrm{~Hz}$ because of the slow convergence rate of the parallel iterative solver at higher frequencies. This is a detail that will be addressed in the future.

\section{Notes on Computational Aspects}

Some details and observations about the computational aspects of this work are presented here.

The finest mesh for the muffler is composed of 153,319 nodes and 107,727 elements.and has a maximum element size of $1 \mathrm{~cm}$. Since each node has two degrees of freedom, due to complex variables, the resulting matrix has 306,638 degrees of freedom. This requires $1,419 \mathrm{Mb}$ of memory for our code to run on one processor. The serial conjugate gradient solver with ILU preconditioner necessitates 271 iterations to solve the system with an accuracy of 1.0e-7 and for a frequency of $200 \mathrm{~Hz}$. 
The finest mesh for the silencer has a maximum element size of $3 \mathrm{~cm}$ and is composed of 180,412 nodes and 114,618 elements. The global system has 360,824 degrees of freedom. The computational domain for the silencer was divided into six subdomains to be solved by six processors in parallel. The partitioning was accomplished such that each parallel baffle is located entirely in one subdomain to improve the convergence rate. Even though this strategy is contradictory with achieving the best load balance, it seems necessary for convergence of the parallel solver to occur. This is due to the use of a localized preconditioner [36]. The maximum memory requirement for each processor is $641 \mathrm{Mb}$. The parallel conjugate gradient solver with localized ILU preconditioner needs 5,272 iterations to solve the system with an accuracy of 1.0e-7 and for a frequency of $200 \mathrm{~Hz}$.

It has been observed that introducing discontinuities in the domain, such as porous media and perforated plates, has a significant negative impact on the convergence rate particularly for a parallel solver. This impact has not yet been quantified or studied closely in this work.

\section{CONClusion}

Successful three-dimensional finite element modeling of a packed muffler and a parallel baffle silencer demonstrate the ability of the FEM in modeling acoustic wave propagation in a non-homogeneous domain. This encourages extending the application of the FEM to more complex cases where other methods have failed to do so accurately. These results also motivate the development of more efficient computational methods, such as the spectral element method (SEM) and more efficient preconditioned iterative solvers. By doing so, it will be possible to address wider frequency ranges, as this 3-D FEM is still expensive regarding the computational cost for solving high frequency acoustic fields. A recent two-dimensional study by the authors of this report demonstrate that a significant gain can be achieved, both in terms of the degrees of freedom and CPU time, by undertaking p-extension, which involves increasing the order of the approximation, as opposed to h-extension, which involves reducing the element size [27]. This confirms the predictions by dispersion analysis [1,31].

To further improve the accuracy of the modeling results, the mathematical model should be improved. For instance, the FEM has potential to include the effects of the mean flow and temperature gradient in the domain and more accurate empirical relations for modeling the porous media and perforated plates.

\section{ACKNOWLEDGMENT}

This work was supported by Engine Control Systems inc. and by the Natural Sciences and Engineering Research Council of Canada (NSERC). The first author would like to thank his colleague, Zakia Sultana, for her help in implementing the parallel solver. 


\section{BibLIOGRAPHY}

[1] Ihlenburg, F., Babuska, I., and Sauter, S., Reliablity of finite element method for the numerical computation of waves, Advances in Engineering Software 28, 417-424, 1997.

[2] Ihlenburg, F., The medium-frequency range in computational acoustics: practical and numerical aspects, J. Comput. Acoust. 11(2), 175-193, 2003.

[3] Farhat, C., Wiedemann-Goiran, P., and Tezaur, R., A discontinuous Galerkin method with plane waves and Lagrange multipliers for the solution of short wave exterior Helmholtz problems on unstructured meshes, Wave Motion 39, 307-317, 2004.

[4] Harari, I., and Magoules, F., Numerical investigations of stablized finite element computations for acoustics, Wave Motion 39, 339-349, 2004.

[5] Koike, T., Wada, H., and Kobayashi, T., Modeling of the human middle ear using the finite-element method, J. Acoust. Soc. Am. 111(3), 1306-1317, 2002.

[6] Tezaur, R., Macedo, A., Farhat, C., and Djellouli, R., Three-dimensional finite element calculations in acoustic scattering using arbitrarily shaped convex artificial boundary, Int. J. Numer. Meth. Engng 53, 1461-1476, 2002.

[7] Lee, F. - C., and Chen, W. -H., On the acoustic absorption of multi-layer absorbers with different inner structures, J. Sound Vib. 259(4), 761-777, 2003.

[8] Munjal, M. L., Analysis and design of mufflers-an overview of research at Indian Institute of Science, J. Sound Vib. 211(3), 425-433, 1998.

[9] Bilawchuk, S., and Fyfe, K. R., Comparison and implementation of the various numerical method used for calculating transmission loss in silencer systems, Appl. Acoust. 64, 903-916, 2003.

[10] Wu, T. W., Cheng, C. Y. R., and Zhang, P., A direct mixed-body boundary element method for packed silencers, J. Acoust. Soc. Am. 111(6), 2566-2572, 2002.

[11] Munjal, M. L., Acoustics of ducts and ufflers, Wiley-Interscience, New York, 1987.

[12] Wu, T. W., and Wan, G. C., Muffler performance studies using a direct mixed-body boundary element method and a three-point method for evaluating transmission loss, ASME Trans., J. Vib. Acoust. 118, 479-484, 1996.

[13] Sullivan, J. W., and Crocker, M. J., Analysis of concentric-tube resonators having unpartitioned cavities, J. Acoust. Soc. Am., 64, 207-215, 1978. 
[14] Delany, M. E., and Bazley, E. N., Acoustical properties of fibrous materials, Appl. Acoust. 3, 105-116, 1970.

[15] Allard, J. F., Propagation of sound in porous media, Elsevier Applied Science, London, 1993.

[16] Utsuno, H., Tanak, T., Fujikawa, T., and Seybert, A. F., Transfer function method for measuring characteristic impedance and propagation constant of porous materials, J. Acoust. Soc. Am. 86, 637-643, 1989.

[17] Beranek, L. L., and Vér, I. L., ed., Noise and vibration control engineering, WileyInterscience, New York, 1992.

[18] Harris, C. M., ed. Handbook of noise control, McGraw-Hill, New York, 1957.

[19] Sullivan, J. W., A method of modeling perforated tube muffler components, I. Theory, J. Acoust. Soc. Am. 66, 772-778, 1979.

[20] Sullivan, J. W., A method of modeling perforated tube muffler components, II. Applications, J. Acoust. Soc. Am. 66, 779-788, 1979.

[21] Munjal, M. L., and Prasad, M. G., On plane wave propagation in a uniform pipe in the presence of a mean flow and a temperature gradient, J. Acoust. Soc. Am. 80, 1501-1506, 1986.

[22] Peat, K. S., The transfer matrix of a uniform duct with a linear temperature gradient, J. Sound Vib. 123, 43-53, 1988.

[23] Harari, I., and Hughes, T. J. R., A cost comparison of boundary element and finite element methods for problems of time-harmonic acoustics, Comput. Meth. Appl, Mech. Eng. 97, 77-102, 1992.

[24] K. Nakajima and H. Okuda, Parallel Iterative Solvers with Localized ILU Preconditioning for Unstructured Grids on Workstation Clusters, Int. J. Comput. Fluid Dyn. 12, 315-322, 1999.

[25] Gropp, W., Lusk, E., and Skjellum, A., Using MPI: portable parallel programming with the Message-Passing Interface, MIT Press, Cambridge, Massachusettes, 1994.

[26] Stroud, A. H., A fifth degree integration formula for the $n$-simplex, SIAM J. Numer. Anal. 6, 90-98, 1969.

[27] Mehdizadeh, O. Z. and Paraschivoiu, M., Investigation of a two-dimensional spectral element method for Helmhotz's equation, J. Comput. Phys. 189, 111-129, 2003. 
[28] Kechroud, R., Soulaimani, A., Saad, Y., and Gowada, S., Preconditioning Techniques for the Solution of the Helmholtz Equation by the Finite Element Method, Math. Comput. Simul. 65, 303-321, 2004.

[29] Mazzia, A., and Pini, G., Numerical Performance of Preconditioning Techniques for the Solution of Complex Sparse Linear Systems, Commun. Numer. Meth. Engng. 19, 37-48, 2003.

[30] Malhotra, M., Freund, R. W., Pinsky, P. M., Iterative Solution of Multiple Radiation and Scattering Problems in Structural Acoustics Using a Block QuasiMinimal Residual Algorithm, Comput. Methods Appl. Mech. Engrg. 146, 173-196, 1997.

[31] Thompson, L. L., and Pinsky, P. M., Complex Wavenumber Fourier Analysis of the P-Version Finite Element Method, Comput. Mech. 13, 255-275, 1994.

[32] Dey, S., Evaluation of p-FEM Approximations for Mid-Frequency ElastoAcoustics, J. Comput. Acoust. 11(2), 195-225, 2003.

[33] Kardestuncer, H., Finite Element Handbook, McGraw-Hill, New York, 1987.

[34] Kikuchi, N., Finite Element Methods in Mechanics, Cambridge University Press, New York, 1986.

[35] Zienkiewicz, O. C., The Finite Element Method, 5th Edition, ButterworthHeinemann, Oxford, 2000.

[36] Wang, K., Kim, S. -B., Zhang, J., Nakajima, K., and Okuda, H., Global and Localized Parallel Preconditioning Techniques for Large Scale Solid Earth Simulations, Future Generation Computer Systems 19(4), 443-456, 2003. 